politischen Philosophie zuläuft und sie ist es auch, die diese kleine Schrift zu einem so wichtigen Beitrag in der aktuellen Auseinandersetzung über das angemessene Verständnis von politischer Theorie bzw. Philosophie und Kritik macht: Indem Geuss eine ideologiekritische, realistische Machtanalyse vorschlägt (75-81), die keineswegs die Beziehung des Politischen zur Normativität dementiert, die aber energisch bestreitet, dass Normativitätsbegründung das Kerngeschäft einer kritischen politischen Philosophie und Theorie ausmacht, unternimmt er einen schlagkräftigen Versuch, einer Form kritischen politischen Denkens jenseits des kantianischen Begründungsgebots den Weg zu bahnen.

Oliver Flügel-Martinsen

\section{Honneth, Axel. Das Ich im Wir. Studien} zur Anerkennungstheorie. Berlin. Suhrkamp 2010. 308 Seiten. $12 €$.

An der Umwandlung der „Anerkennung " von einem Gegenstand oder einem Themenfeld sozialwissenschaftlicher oder sozialphilosophischer Forschung in den Namen einer eigenständigen theoretischen Richtung hat neben anderen Axel Honneth erheblichen Anteil. Er legt nun ergänzende, klärende und vertiefende Untersuchungen vor, die den explikativen Anspruch der Anerkennungstheorie weiter untermauern sollen. In vier Abschnitten unternimmt Honneth dies a) über die Rückvergewisserung in der Hegelschen Philosophie des Geistes, der er die strukturellen Schemata seiner Theorie entlehnt, b) über systematische Auseinandersetzungen mit gerechtigkeitstheoretischen Problemen und Positionen, c) über soziologisch-deskriptive Analysen und d) über psychoanalytische Ergänzungen.

Als Leitfaden der untereinander methodisch wie thematisch recht heterogenen Beiträgen wird man die theoriepolitische These ausmachen können, dass eine Umstellung der sozialphilosophischen Theoriebildung von der Orientierung an Gleichheit auf die an Anerkennung eine Wende von monistischen auf pluralistische Ansätze bedeutet (167). Vorbereitet wird diese These in den beiden Hegel-Aufsätzen, die der bereits bekannten Verwendung, die Honneth von Hegel macht, nicht viel Neues hinzufügen; entfaltet wird sie vor allem in dem programmatischen Aufsatz „Das Gewebe der Gerechtigkeit. Über die Grenzen des zeitgenössischen Prozeduralismus“. In den gegenwärtig dominanten Gerechtigkeitstheorien, die ihre liberale Stoßrichtung vor allem einem kantischen Erbe verdanken, werden zunächst drei relevante Bestandteile kritisch gesichtet: ein prozeduralistisches Grundschema, die Orientierung an der Verteilungsgerechtigkeit und ihre Staatsfixierung. Der Ausgangspunkt bei der Überzeugung, Gerechtigkeit werde in erster Linie über die Gewährung eines allen gemeinsamen Spielraums für die Verfolgung individueller Präferenzen gewährleistet, sorgt dafür, dass die drei Aspekte dort an ihre Grenzen kommen, wo das individuelle Nutzenkalkül das Gerechtigkeitsempfinden nicht zur Gänze abzudecken vermag beziehungsweise mit diesem in Widerspruch gerät. So muss der Prozeduralismus Autonomie schon voraussetzen, obwohl sie doch erst am Ende des Abgleichs von Interessen als Resultat sich ergeben soll. So schließt das Distributionsparadigma all jene Aspekte der Gerechtigkeit aus der Betrachtung aus, die sich 
nicht als verteilbare individuelle Güter betrachten lassen. Und so blendet die Fixierung auf den Staat als Distributor der prozedualistisch festgelegten Anteile all jene sozialen und politischen Akteure der Zivilgesellschaft aus, die ebenfalls zur Gerechtigkeit etwas beizutragen hätten. Ex negativo ergibt sich das Erfordernis, nicht ein Leitkriterium der Gerechtigkeit, nämlich die distributionstheoretisch verstandene Gleichheit, in Geltung zu bringen, sondern der Pluralität der lebensweltlichen Dimensionen der Gerechtigkeit jeweils unterschiedliche normative Leitmedien zuzuweisen. Dies mündet in die Überzeugung, dass je „nach Sozialsphäre, der sie sich zuwendet, [eine pluralistische Gerechtigkeitstheorie, Th.B.] [...] den moralischen Gesichtspunkt entweder der deliberativen Gleichheit, der Bedürfnisgerechtigkeit oder der Leistungsgerechtigkeit hervorzuheben und zu verstärken " hat. (76) Unschwer zu erkennen ist hier, dass diese bereits aus Honneths früheren Werken bekannte These an die Hegel-Lektüren anschließt und dessen drei Sphären der moralischen, persönlichen und sozialen Freiheit aktualisiert. Mit Hegel folgt aus diesem Ansatz auch, dass er als „rekonstruktiv angelegte Gerechtigkeitstheorie" (73) angelegt wird, das heißt, dass kein unparteiischer und letztlich ahistorischer idealer Standpunkt konstruiert wird, sondern aus dem gegebenen Stand der institutionellen Verfasstheit der Gesellschaft und den moralischen Intuitionen ihrer Mitglieder jene normativen Bestandteile rekonstruiert werden, die der Philosoph dann ordnet (wie es in der Monographie „Das Recht der Freiheit“, Berlin 2011, ausführlich vorgeführt wird).

Ein solch rekonstruktives Verfahren, das sich eher an Hegel statt an Kant anlehnt, ist stärker als letzterer darauf angewiesen, sich von der Empirie, und das heißt hier: von sozialwissenschaftlichen Forschungen, informieren $\mathrm{zu}$ lassen. So wird die pluralistische Theorie der Gerechtigkeit von David Miller gelobt (158ff.), Paradoxien der kapitalistischen Modernisierung beziehungsweise der Individualisierung (als deren Teil) entfaltet und der Versuch gemacht, den von Philosophen üblicherweise vernachlässigten Arbeitsbegriff dadurch normativ aufzuladen, dass das Feld der Erwerbsarbeit als notwendiger Bestandteil umfassender personaler Anerkennung betrachtet wird.

In den sich auf sozialwissenschaftliche Expertise stützenden Untersuchungen $\mathrm{zu}$ den genannten gesellschaftlichen Paradoxien, die dadurch gekennzeichnet sind, dass sich ehemals emanzipatorische Forderungen nun in Imperative sozialen oder ökonomischen Zwangs verwandelt haben, treten jedoch auch die Grenzen der Anerkennungstheorie zu Tage, wie Honneth sie konzipiert. Denn man könnte doch Formen der Anerkennung ins Feld führen, die gerade nicht zur Bildung oder Stärkung der individuellen Autonomie beitragen, sondern nur der „Erzeugung von systemkonformen Einstellungen“ (103) dienen. An Hand des Beispiels des Strukturwandels der Arbeitsorganisation führt Honneth zunächst eine solche Form der ideologischen oder "normalisierenden Anerkennung “ (121) vor. Die Rede vom kreativen „Arbeitskraftunternehmer“, der sich gewissermaßen in Ausübung seiner ökonomischen Pflicht gleich selbst mit verwirklicht, dient offenkundig den Interessen des flexibilisierten Kapitalismus. Als solcher anerkannt zu werden oder sich anerkennen zu lassen, kann daher kaum in das normative Muster 
Honneths eingebaut werden. Es scheint sich hier eher um eine Verkennung statt um eine Anerkennung zu handeln. Doch Honneths robuster Fortschrittskonzeption gelingt es, auch noch dieser Form der Funktionalisierung von Selbstverwirklichungsimperativen einen normativen Impuls abzugewinnen, indem er behauptet, die Rede vom „Arbeitskraftunternehmer" sei nicht per se „defizitär oder irrational“, sondern genüge nur „nicht den materiellen Anforderungen, an denen sich die Glaubwürdigkeit der gerechtfertigten Anerkennungen erst vollständig bemessen würde" (129f.). Mit anderen Worten: Die Anrufung des kreativen „Arbeitskraftunternehmers" ist keine ideologische Indienstnahme ehemals emanzipatorischer Diskurse („Sei Du selbst!"), sondern verweist normativ auf eine noch ausstehende Transformation der Produktionsbedingungen.

Der rekonstruktive Charakter der Honnethschen Theorie der Anerkennung scheint hier an seine Grenzen zu stoßen. Denn entweder endet die empirische Erhebung sozialer Transformationsprozesse in Paradoxien, die normativ nicht eindeutig zu bewerten sind, oder die Empirie wird nach Maßgabe der normativen Erwartungen der Theorie zugeschnitten. Schließlich steht hinter dem begrüßenswerten Aufgreifen empirischer Wirklichkeiten, die Philosophen allzu gerne vernachlässigen, das normative Telos eines autonomen Subjekts, das durch intersubjektiv vermittelte Anerkennung zu einer versöhnten Identität gelangt. Ist aber die (zumindest in diesem Werk) nicht näher spezifizierte Autonomie das normative Ideal, so sind dann doch die Differenzen zu liberalen Theorien geringer als ursprünglich von Honneth in Aussicht gestellt. Pluralistische und monis- tische Theorien der Gerechtigkeit wären dann nicht so sehr zwei grundverschiedene Paradigmen als zwei Alternativen auf dem Weg zum klassisch philosophischen Topos des autonomen Subjekts.

Thomas Bedorf

\section{Politisches System der Bundesrepublik Deutschland}

Hassel, Anke, und Christof Schiller. Der Fall Hartz IV. Wie es zur Agenda 2010 kam und wie es weitergeht. Frankfurt am Main/New York. Campus Verlag 2010. 348 Seiten. 24,90€.

Unter politischen Akteuren und Beobachtern des politischen Geschehens herrscht weitgehende Einigkeit, dass systemische Veränderungen in Deutschland kaum erfolgreich ins Werk zu setzen sind. Die von Fritz Scharpf beschriebene "strukturverändernde Politik“ steht unter den Zugkräften interessengeleiteter Parteipolitik, Verteilungsaspekten und föderalen Verflechtungen. Damit bleiben politische Reformen stets nur Stückwerk - und der Nachwelt als Status quo orientierte Anpassungen ohne visionären Veränderungsanspruch erhalten.

Mit ihrer umfangreichen Studie über die Agenda 2010 und die Entstehung von Hartz IV untersuchen Anke Hassel und Christof Schiller eines der zentralen Handlungsfelder der rot-grünen Bundesregierung. In der Einschätzung der Autoren ist Hartz IV „eine der umstrittensten Reformen der Nachkriegszeit" (9); gleichzeitig gibt die Entstehungsgeschichte der Hartz-Gesetze Aufschluss über „die Dynamik politischer Entscheidungsverfahren " (11). Hassel und Schiller zeigen, „dass die 